\title{
Epstein-Barr virus expression in plasma cell neoplasms and its association with plasmablastic morphologic features
}

\author{
Whitney Pasch • William Wu • Dylan Bach • \\ Nagesh Rao $\cdot$ Xiaohui Zhao $\cdot$ Sherif A. Rezk
}

Received: 4 October 2012 / Accepted: 18 March 2013 /Published online: 2 April 2013

(C) Springer-Verlag Berlin Heidelberg 2013

\begin{abstract}
Plasma cell neoplasms include plasma cell myeloma, plasmacytoma, primary amyloidosis, and light and heavy chain deposition diseases. Epstein-Barr virus (EBV) expression has rarely been reported to be associated with plasma cell neoplasms except for patients with a previous history of transplantation or severe immunodeficiency. In contrast, EBV expression has been reported in the majority of plasmablastic lymphoma, a subtype of diffuse large B cell lymphoma. We report three cases with a plasma cell neoplasm (two plasma cell myelomas and one case of plasmacytoma), where EBV infection was diffusely detected by in situ hybridization. All three patients were under the age of 40 years, and one of the three patients was an HIVpositive individual while the other two patients did not have any identifiable source for immunosuppression. All three cases showed multifocal plasmablastic features, which is concordant with the most recent reports about the possible association of EBV expression with plasmablastic morphologic features and increased proliferative activity.
\end{abstract}

Keywords Epstein-Barr virus · Plasma cell neoplasms · Plasmablastic features

W. Pasch $\cdot$ W. Wu $\cdot$ D. Bach $\cdot$ X. Zhao $\cdot$ S. A. Rezk Department of Pathology \& Laboratory Medicine, University of California, Irvine (UCI), Irvine, CA, USA

N. Rao

Department of Pathology \& Laboratory Medicine, University of California, Los Angeles (UCLA), Los Angeles, CA, USA

S. A. Rezk $(\bowtie)$

Department of Pathology, University of California Irvine Medical Center (UCI), 101 The City Drive, Bldg 54, Rm 4702,

Orange, CA 92867, USA

e-mail: srezk@uci.edu

\section{Introduction}

Plasma cell neoplasms encompass a spectrum of plasma cell disorders that include plasma cell myeloma, plasmacytoma, primary amyloidosis, and light and heavy chain deposition diseases $[1,2]$. They are characterized by a monoclonal proliferation of immunoglobulin-producing plasma cells that represent post-germinal center, terminally differentiated B lymphocytes $[1,2]$. Two types of plasmacytomas are recognized in the 2008 World Health Organization (WHO) classification: solitary plasmacytoma of bone (osseous plasmacytoma) and extraosseous plasmacytoma (extramedullary plasmacytoma) $[1,2]$. Solitary plasmacytoma of bone usually presents as a lytic lesion involving the axial skeleton and frequently progresses to plasma cell myeloma in up to twothirds of the patients, while the extramedullary type has a predilection for the upper aerodigestive tract and has a more indolent clinical behavior with only $15 \%$ progressing to an overt plasma cell myeloma [1,2].

True plasma cell neoplasms have been rarely reported to be associated with Epstein-Barr virus (EBV) expression. Plasmablastic lymphoma, a subtype of diffuse large B cell lymphoma with near-identical immunophenotype to plasma cell neoplasms, has been reported to show EBV expression in about two-thirds of the cases and near $100 \%$ expression in the oral mucosa subtype $[3,4]$. Post-transplant patients and severely immunocompromised patients, especially patients with established HIV infection, have been reported to have slightly increased incidence for EBV-positive plasma cell neoplasms although the cytomorphologic features of the neoplastic cells in such cases have been mainly described as plasmablastic or anaplastic [2, 5]. Plasmablastic cytomorphologic features are defined as plasma cells having larger concentrically placed nucleus with little or no cytoplasmic hof and exhibiting open or coarse chromatin, prominent nucleoli (usually central), and 
less abundant cytoplasm [6]. The anaplastic cytomorphologic features are defined as plasma cells with a hyperchromatic nuclei and more pleomorphic appearance with irregular nuclear and cytoplasmic contours [7]. Plasma cell myeloma patients with plasmablastic or anaplastic morphology tend to have a more aggressive clinical course and are generally considered to carry a more guarded prognosis [2]. We report three cases with a plasma cell neoplasm (two plasma cell myelomas and one case of plasmacytoma), where EBV expression was diffusely detected by in situ hybridization. All three patients were under the age of 40 years, and one of the three patients was an HIV-positive individual while the other two patients did not have any identifiable source for immunosuppression.

\section{Clinical history, methods, and results}

\section{Case 1}

A 39-year-old Hispanic male presented to the emergency room in early 2012 with a 2-month history of abdominal pain radiating to his back and with leg numbness for 2 days prior to presenting to the hospital. His past medical history was unremarkable except for hypothyroidism. A computed tomography (CT) and magnetic resonance imaging bone scans revealed lytic bony lesions at the cervical, thoracic, and lumbar spine as well as at the skull and left rib. A biopsy of the thoracic lesion revealed sheets of kappa-restricted plasma cells that showed strong expression of EBV by in situ hybridization (EBV-encoded RNA (EBER)) with a small subset expressing EBV-latent membrane protein 1 (LMP-1) by immunohistochemistry. The neoplastic plasma cells showed lack of expression for the B cell markers CD20 and PAX-5 as well as for cyclin D1, CD56, and human herpesvirus 8 (HHV-8). Focal plasmablastic features were noted on morphologic examination and an overall low Ki-67 proliferative activity was noted (10\%) although the plasmablastic areas showed slightly increased proliferative activity. The intensity of staining and percentage of EBERpositive cells were similar in both areas. Subsequent biopsies from the cervical spine and a right parietal mass showed an identical process with a similar immunophenotype. A bone marrow biopsy was performed and showed a slightly hypocellular marrow with active trilineage hematopoiesis and no evidence of involvement by a plasma cell neoplasm. EBER stain showed negative results when performed on the bone marrow clot biopsy sections. EBV serology testing was not performed. Cytogenetic studies performed on the bone marrow aspirate showed a normal male karyotype. Fluorescence in situ hybridization (FISH) with the dualcolor MYC "split" probe was unsuccessful due to the decalcification method used to fix the cells but hinders DNA probe hybridization and the visualization of the fluorescent signals. HIV testing by serology showed negative results. Serum protein electrophoresis showed a prominent betarestricted band quantitated at $1.29 \mathrm{~g} / \mathrm{dl}$ and was identified as an IgA kappa paraprotein by immunofixation. Urine electrophoresis and immunofixation showed free kappa light chains. Laboratory tests showed mild leukopenia, hypercalcemia, elevated serum free light chains, and elevated IgA levels. The patient also had mild anemia and mild thrombocytopenia. The patient's mental status rapidly declined and a lumbar puncture was performed, which revealed involvement of the cerebrospinal fluid by the neoplastic plasma cells having the same morphological picture as the previous biopsy. The patient is currently receiving radiation therapy and has shown relative overall improvement despite the persistence of the monoclonal paraproteins in his serum.

\section{Case 2}

A 39-year-old Hispanic female who was diagnosed with HIV in 2000 presented to our institution in early 2008 with abdominal pain and distension for 5 days prior. Her CD4+ T cell count at presentation was $47 \mathrm{cells} / \mu \mathrm{L}$. Multiple diffuse bony lesions were visualized on skeletal survey in addition to several ovarian and liver masses. An ultrasound-guided fine needle aspiration biopsy of the liver mass showed sheets of kappa-restricted plasma cells that express CD138 and CD56 by immunohistochemistry. The neoplastic plasma cells were negative for EBV-LMP-1, HHV-8, and cyclin D1 stains. By in situ hybridization, the neoplastic plasma cells were EBV (EBER) positive. EBV serology testing was not performed. Serum protein electrophoresis showed a band with restricted mobility in the fast gamma region that was quantitated at $3.25 \mathrm{~g} / \mathrm{dl}$. Immunofixation revealed an IgA kappa and IgG kappa biclonal gammopathy. Her urine protein electrophoresis showed free kappa light chains. A bone marrow biopsy from bilateral iliac crests showed 70-80\% marrow involvement by the neoplastic myeloma cells that exhibited anaplastic and plasmablastic features and demonstrated CD138 and kappa light chain expression by immunohistochemistry and flow cytometry. A subset of the neoplastic plasma cells within the bone marrow was positive for EBER stain. Cytogenetic studies performed on the bone marrow aspirate showed a normal female karyotype. FISH with dual-color MYC split probe did not yield unambiguous fluorescent signals. The patient succumbed to the disease 2 months later after complications from myeloma.

\section{Case 3}

A 38-year-old Caucasian male presented to our institute in late 2000 with altered mental status and epistaxis. A CT scan 
of the head and neck revealed a lesion in the right nasal cavity extending into the right frontal lobe. A subsequent skeletal survey revealed multiple lytic bony lesions throughout the body. Laboratory testing showed elevated serum BUN and creatinine in addition to normocytic anemia. Testing for HIV-1 and HIV-2 by polymerase chain reaction (PCR) showed negative results. Serum protein electrophoresis showed a band in the fast gamma region that was quantitated at $1.25 \mathrm{~g} / \mathrm{dl}$. Immunofixation showed an $\operatorname{IgG}$ kappa monoclonal paraprotein. A free kappa light chain band was demonstrated in the urine by immunofixation as well. A biopsy of the rapidly growing nasal lesion revealed sheets of kappa-restricted plasma cells that exhibited plasmablastic morphology in many areas although areas of plasma cells with normal morphology were also seen. EBV studies by in situ hybridization (EBER) showed positivity in the vast majority of the neoplastic plasma cells including those with normal and plasmablastic morphologic features. The intensity of staining and percentage of EBER-positive cells were relatively similar in both areas (Fig. 1). The neoplastic cells were negative for EBV-LMP-1, HHV-8, cyclin D1, and CD56 by immunohistochemistry. A bone marrow biopsy of the iliac crest showed a normocellular marrow with active trilineage hematopoiesis and no evidence of involvement by clonal plasma cells. EBER stain showed negative results when performed on the bone marrow clot biopsy sections. Attempts to obtain MYC gene
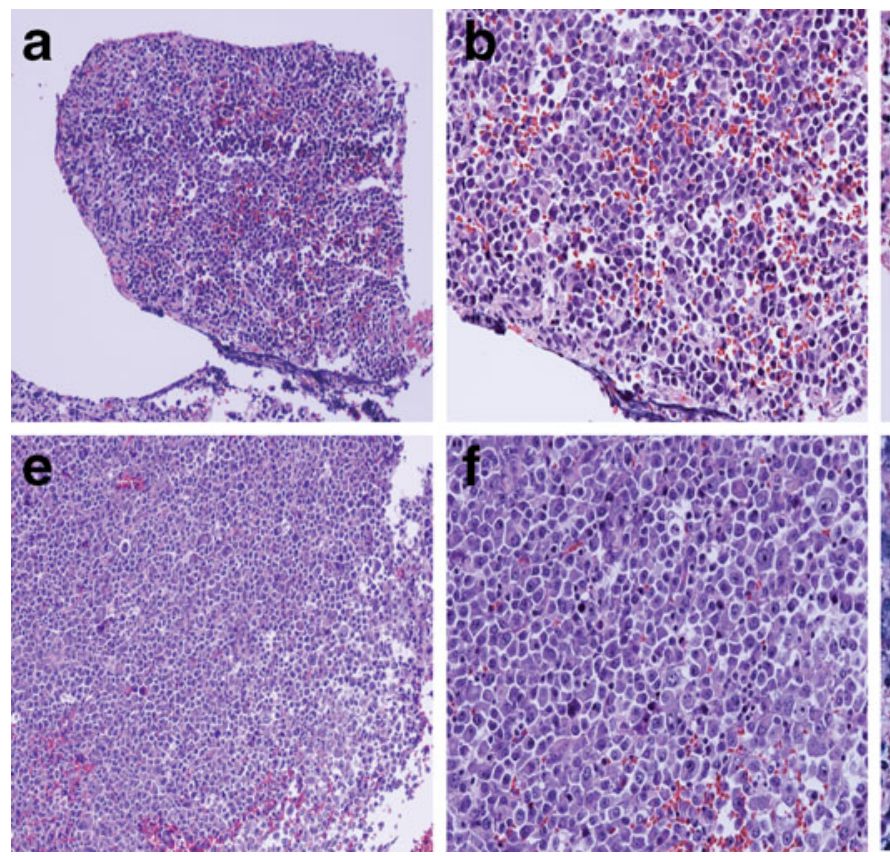

Fig. 1 The composite picture represents two different fields from case 3. The upper half shows sheets of neoplastic plasma cells that show no atypical features. a Low-power magnification $(\times 10)$ of the plasma cells and $\mathbf{b}$ high-power magnification $(\times 40)$. $\mathbf{c}$ EBER expression (EBV by in situ hybridization) $(\times 40)$ and $\mathbf{d} \mathrm{Ki}-67$ expression shows minimal proliferative activity $(\times 40)$. The lower half of the composite picture shows status by dual-color FISH were unsuccessful. The patient underwent treatment with vincristine, doxorubicin, and dexamethasone with pamidronate and radiation therapy but did not show initial response to treatment and was ultimately lost to follow-up and his living status is unknown.

\section{Discussion}

In contrast to plasmablastic lymphomas, true plasma cell neoplasms have been rarely associated with EBV infection although post-transplant patients and severely immunocompromised patients may have slightly increased incidence of having an EBV-positive plasma cell neoplasm $[2,5]$. The distinction of plasmablastic lymphoma from plasma cell myeloma or plasmacytoma with plasmablastic features can be difficult at times and usually depends on clinical correlation with the presence of serum monoclonal paraproteins, bone marrow involvement, and radiographically evident lytic lesions, which are all factors that would favor plasma cell myeloma with plasmablastic features [7, 8]. Although the expression of EBV and a positive HIV status have been historically associated with a diagnosis of plasmablastic lymphoma, other more reliable factors have been postulated to support a diagnosis of plasmablastic lymphoma over myeloma such as markedly increased mitotic activity, presence of numerous apoptotic bodies, and paucity of maturing

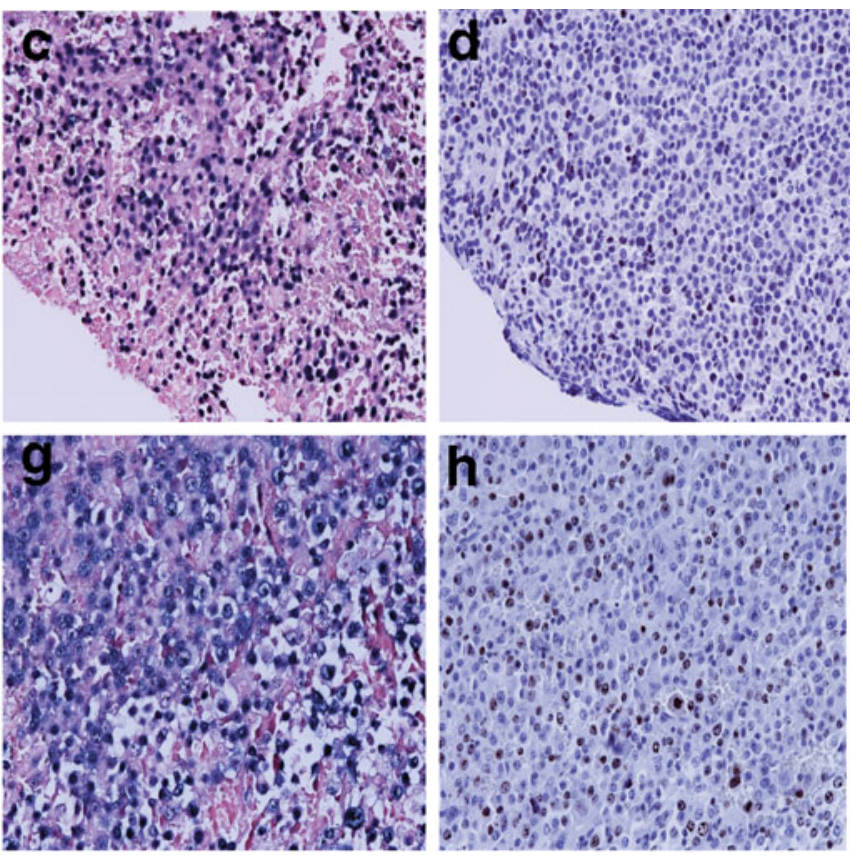

sheets of plasma cells with atypical and plasmablastic features and a higher proliferative activity. e Low-power magnification $(\times 10)$ of the atypical plasma cells and $\mathbf{f}$ high-power magnification $(\times 40)$. g EBER expression $(\times 10)$ and $\mathbf{h}$ Ki-67 expression shows increased proliferative activity than the areas without the plasmablastic features $(\times 40)$ 


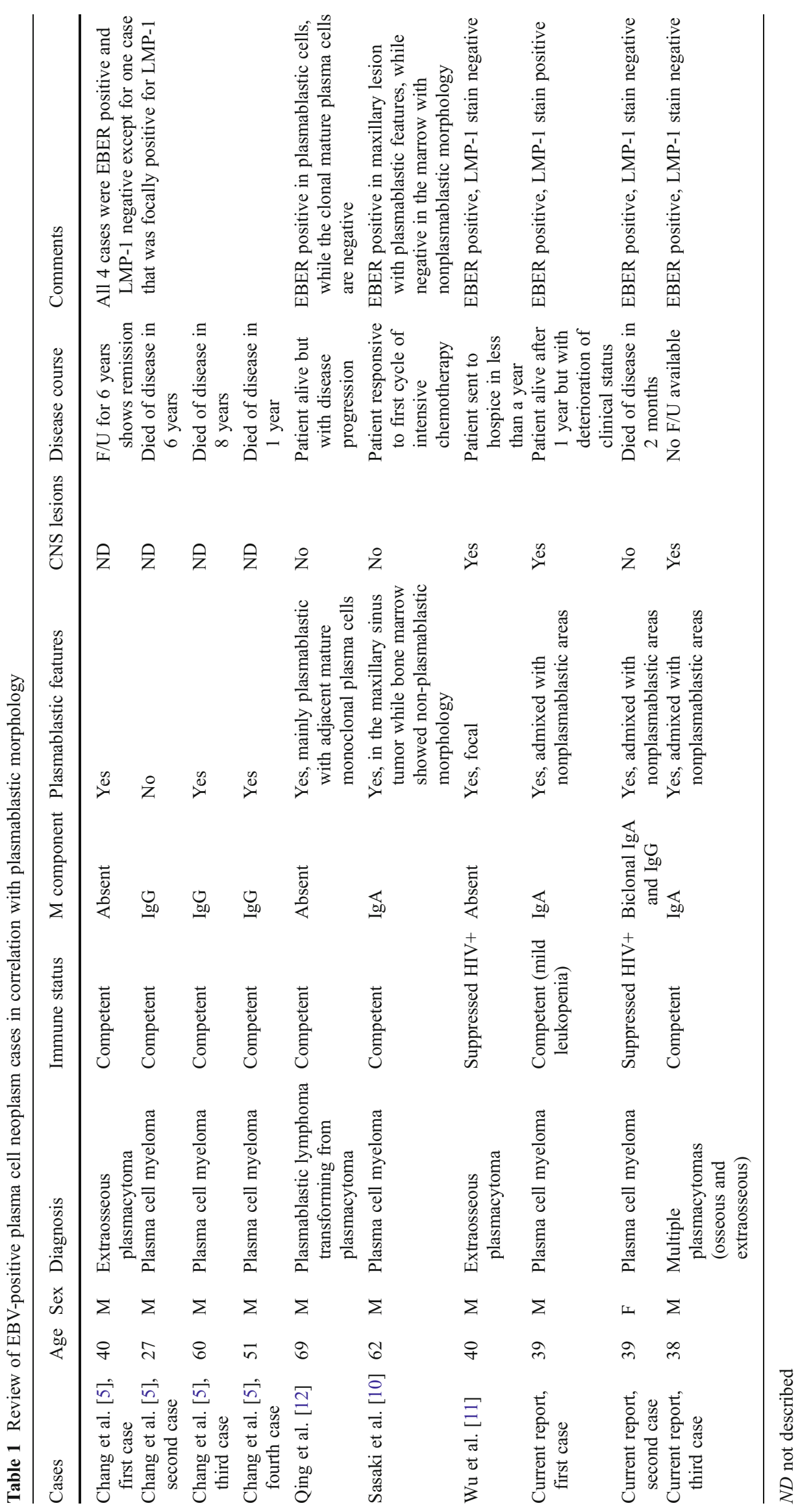


plasma cells [7]. Vega et al. have postulated in their study that EBV expression constitutes the differentiating factor between plasma cell neoplasms and plasmablastic lymphoma, where the latter show EBV expression in the majority of cases [3]; however, others have reported the detection of EBV in extramedullary plasmacytoma of the head and neck in immunocompetent individuals in up to $15 \%$ of all cases [9]. Moreover, other investigators have reported the identification of EBV expression in immunocompetent patients with plasma cell myelomas and plasmacytomas, where the plasma cells in these cases exhibited plasmablastic morphology, and they concluded that the presence of EBV in plasma cell neoplasms is significantly associated with plasmablastic morphologic features suggesting that these tumors may have been driven by EBV to gain the plasmablastic morphologic features and a higher proliferation rate [5, 10-12]. Also, the identification of EBV expression in a subtype of patients with plasmablastic lymphoma that occurred as a result of plasma cell myeloma progression/transformation has been reported [13]. A more recent study has identified EBV expression by PCR in $33 \%$ of bone marrows with plasma cell myeloma compared to only $10 \%$ of normal bone marrow specimens [14], although the results may not be truly representative due to small sample size and the ethnicity of patients.

In healthy individuals who are seropositive for EBV, terminal differentiation of EBV-infected memory B cells into plasma cells has been reported to be associated with viral replication and initiation of the EBV productive (lytic) cycle $[15,16]$. In contrast, individuals with plasmacytoid tumors frequently associated with EBV such as primary effusion lymphoma or plasmablastic lymphoma have been shown to establish a nonproductive (latent) cycle and exhibit a restrictive EBV latency pattern (latency I/II) $[16,17]$. In addition, a strong reduction in plasma cell-associated markers such as CD38 and CD138 and a regression to a less mature $\mathrm{B}$ cell phenotype were observed in the EBVinfected plasmacytoid tumor cells as well as in few plasma cell myeloma cell lines examined in vitro [16]. As such, plasma cell myeloma or plasmacytoma cases that express EBV, whether as a result of an associated immunodeficiency or for unknown reasons, may follow the same path as plasmablastic lymphoma and exhibit a non-productive (latent) cycle, increase proliferation, and exhibit plasmablastic cytologic features starting in focal areas and probably eventually progressing to plasmablastic lymphoma.

Despite the fact that plasma cell neoplasms occur rarely in young individuals with the vast majority of cases occurring over the age of 50 and a median age at diagnosis of 70 years for plasma cell myeloma and 55 years for plasmacytoma (osseous and extraosseous) [2, 18], all three of our patients were under the age of 40 years at diagnosis and all three had presented with a very aggressive clinical course including involvement of the central nervous system (CNS) in two cases. All three patients presented with morphologic evidence of a plasmablastic morphology whether within the majority of cells or within a subset of the neoplastic plasma cells. One patient was HIV positive, while the other two showed no definitive evidence of an associated immunosuppressive state. All three of our cases showed no reduction in CD138 expression and two out of the three cases were negative for EBV-LMP-1 expression by immunohistochemistry but positive for EBER by in situ hybridization, which is suggestive of a restricted EBV latency pattern, probably latency I. Based on our case series and other cases reported in the literature (Table 1), there may be indeed a notable correlation between EBV expression and the presence of plasmablastic morphologic features as well as with increased proliferative activity and a potential aggressive clinical course compared to the relatively more indolent and prolonged clinical course usually seen in EBV-negative plasma cell neoplasm cases, especially plasmacytomas. The EBV role may be through alternate pathway other than the LMP-1 protein-related neoplastic transformation. Further studies with a larger series of cases are needed to validate these findings.

Conflict of interest The authors declare that they have no conflict of interest.

\section{References}

1. Lorsbach RB, His ED, Dogan A, Fend A (2011) Plasma cell myeloma and related neoplasms. Am J Clin Pathol 136(2):168182

2. McKenna RW, Kuehl WM, Grogan TM, Harris NL, Coupland RW (2008) Plasma cell neoplasms. In: Swerdlow SH, Harris NL, Jaffe ES, Pileri SA, Stein H, Thiele J, Vardiman JW (eds) WHO classification of tumors of hematopoietic and lymphoid tissues. IARC, Lyon, pp 200-214

3. Vega F, Chang CC, Medeiros LJ, Udden MM, Cho-Vega JH, Lau CC, Finch CJ, Vilchez RA, McGregor D, Jorgensen JL (2005) Plasmablastic lymphomas and plasmablastic plasma cell myelomas have nearly identical immunophenotypic profiles. Mod Pathol 18(6):806-815

4. Stein H, Campo E (2008) Plasmablastic lymphoma. In: Swerdlow SH, Harris NL, Jaffe ES, Pileri SA, Stein H, Thiele J, Vardiman JW (eds) WHO classification of tumors of hematopoietic and lymphoid tissues. IARC, Lyon, pp 256-258

5. Chang ST, Liao YL, Lu CL, Chuang SS, Li CY (2007) Plasmablastic cytomorphologic features in plasma cell neoplasms in immunocompetent patients are significantly associated with EBV. Am J Clin Pathol 128(2):339-344

6. Greipp PR, Raymond NM, Kyle RA, O'Fallon WM (1985) Multiple myeloma: significance of plasmablastic subtype in morphological classification. Blood 65(2):305-310 
7. Dong HY, Scadden DT, de Leval L, Tang Z, Isaacson PG, Harris NL (2005) Plasmablastic lymphoma in HIV-positive patients: an aggressive Epstein-Barr virus-associated extramedullary plasmacytic neoplasm. Am J Surg Pathol 29(12):1633-1641

8. Taddesse-Heath L, Meloni-Ehrig A, Scheerle J, Kelly JC, Jaffe ES (2010) Plasmablastic lymphoma with MYC translocation: evidence for a common pathway in the generation of plasmablastic features. Mod Pathol 23(7):991-999

9. Aguilera NS, Kapadia SB, Nalesnik MA, Swerdlow SH (1995) Extramedullary plasmacytoma of the head and neck: use of paraffin sections to assess clonality with in situ hybridization, growth fraction, and the presence of Epstein-Barr virus. Mod Pathol 8(5):503-508

10. Sasaki S, Hashimoto K, Nakatsuka S, Hasegawa M, Nakano T, Nagata S, Kanakura Y, Hayashi N (2011) Plasmablastic extramedullary plasmacytoma associated with Epstein-Barr virus arising in an immunocompetent patient with multiple myeloma. Intern Med 50(21):2615-2620

11. Wu W, Pasch W, Zhao X, Rezk SA (2012) Extraosseous plasmacytoma with an aggressive course occurring solely in the CNS. Neuropathology. doi:10.1007/s00277-013-1722-4

12. Qing X, Sun N, Chang E, French S, Ji P, Yue C (2011) Plasmablastic lymphoma may occur as a high-grade transformation from plasmacytoma. Exp Mol Pathol 90(1):85-90
13. Colomo L, Loong F, Rives S, Pittaluga S, Martinez A, LopezGuillermo A, Ojanguren J, Romagosa V, Jaffe ES, Campo E (2004) Diffuse large B-cell lymphomas with plasmablastic differentiation represent a heterogeneous group of disease entities. Am J Surg Pathol 28(6):736-747

14. Sadeghian MH, Ayatollahi H, Keramati MR, Memar B, Jamedar SA, Avval MM, Sheikhi M, Shaghayegh G (2011) The association of Epstein-Barr virus infection with multiple myeloma. Indian $\mathrm{J}$ Pathol Microbiol 54(4):720-724

15. Anastasiadou E, Vaeth S, Cuomo L, Boccellato F, Vincenti S, Cirone M, Presutti C, Junker S, Winberg G, Frati L, Wade PA, Faggioni A, Trivedi P (2009) Epstein-Barr virus infection leads to partial phenotypic reversion of terminally differentiated malignant B cells. Cancer Lett 284(2):165-174

16. Laichalk LL, Thorley-Lawson DA (2005) Terminal differentiation into plasma cells initiates the replicative cycle of Epstein-Barr virus in vivo. J Virol 79(2):1296-1307

17. Rezk SA, Weiss LM (2007) Epstein-Barr virus-associated lymphoproliferative disorders. Hum Pathol 38(9):1293-1304

18. International Myeloma Working Group (2003) Criteria for the classification of monoclonal gammopathies, multiple myeloma and related disorders: a report of the International Myeloma Working Group. Br J Haematol 121(5):749-757 